\title{
SCALABLE PATTERN RECOGNITION AND REAL TIME TRACKING OF MOVING OBJECTS
}

\author{
Dipak Pudasaini \\ Abdolreza Abhari \\ Department of Computer Science \\ Ryerson University \\ 350 Victoria Street, Toronto, ON, Canada \\ \{dpudasaini,aabhari\}@ryerson.ca
}

\begin{abstract}
This paper proposed a new approach for object tracking and pattern recognition of moving objects during real time video streaming. This approach uses motion based multi-object movement techniques for tracking the objects. Moreover, Spectral clustering with Dynamic Time Warping (DTW) and Naïve Bayes method are used for pattern recognition of tracked objects. This system tracks the moving objects collected as a batch of videos then the pattern recognition technique uses for analyzing vehicles movement to determine normal or abnormal behavior. This paper proposes the tracking algorithm for all moving objects and pattern recognition for only moving vehicles. The performance of tracking trajectories is calculated by finding recall and precision values, which are greater than $95 \%$. The experimental result shows that Naïve Bayes is better than spectral clustering for the classification of vehicle trajectories that conforms Naïve Bayes is an effective tool to scale the pattern recognition of moving vehicles.
\end{abstract}

Keywords: object tracking, pattern recognition, multi-object movement, spectral clustering, Naïve Bayes.

\section{INTRODUCTION}

Trajectory pattern recognition is becoming an important research area nowadays. Large numbers of trajectory images are publicly available from photos, web sites, videos etc. The images require a large amount of memories and more computing power for processing. The trajectory pattern recognition and classification are interesting research areas for several applications such as social media, traffics, film industries, earth quick measurement and others (Mazimpaka and Timpf 2016).

It is a challenging task to perform real time and accurate trajectory prediction of moving objects. Many methods are improving and trying to classify the trajectory images. We can see the trajectory images in videos, movies, video surveillance system etc. Videos are a sequence of images; each sequence is called frame. The image processing techniques are applied for individual frames. The content of two consecutive frames are generally similar. Object detection in trajectory images involves verifying the presence of an object in the image sequence and locating it for detection.

The process of object tracking analysis is to input the videos into the object tracking system then determine the number of moving objects, their sizes, and positions. There are different types of object tracking methods used to track objects such as color based, motion-based, shape-based and more (Yilmaz, Javed and Shah 2006). The proposed method in this paper used motion-based tracking of the objects. The existing motion-based methods for tracking the objects are not enough for tracking all the objects in a video file. 


\section{Pudasaini and Abhari}

This proposed method for tracking the objects is more accurate. Trajectory images can be classified using different types of methods such as clustering, Naïve Bayes, Support Vector Machine (SVM), multiclassifiers, Convolutional Neural Network $(\mathrm{CNN})$ and more. It is cleared that SVM, CNN, deep learning and any learning-based method cannot be used in an online manner. These methods need feature extraction, training and parameter tuning phase which should be performed offline or by supervision to be able to build labels and models. The proposed technique in this paper uses clustering and Naïve Bayes methods for pattern recognition. The clustering method does not require training and performs feature extraction automatically. Clustering is an unsupervised machine learning algorithm which is used for scalable data because it can learn the features and labels which are needed for classification without supervision. The Naïve Bayes is a supervised machine learning algorithm that needs features extraction for the classification.

In this paper, we proposed a model for tracking a large number of moving objects and pattern recognition for moving vehicles of videos in the real time video streaming. This technique used a motion-based method for tracking the objects in real time then spectral clustering and Naïve Bayes for pattern recognition. The proposed method can be used for efficiently tracking the moving objects in a video on demand and can be scaled to a large number of videos in social networks.

The rest of the paper is organized as follows: Section 2 presents the literature review of the proposed work. Section 3 presents the details of our proposed approach. The experimental results are explained in Section 4 and Section 5 summarizes the conclusion of this paper.

\section{LITERATURE REVIEW}

The real-time data mining of the sequence of frames for processing trajectories, collected by security camera has been explained (Abhari, Banihashemi and Li 2018). The quality of the object descriptions directly depends upon an object image, color model and the quality of the tracking model. Most visual tracking methods include image input, feature description, context information integration, decision and model update (Yang et al. 2011). Image segmentation partitions the images into distinct regions containing each pixel with similar attributes (Pedro, Antonio and Armand 2007). For color images using Red Green Blue (RGB), space is divided into the number of regular grids, each containing many similar colors (Zhang and $\mathrm{Wu}$ 2012). The color-based object detection and tracking method using simple tools and techniques of computer vision have been proposed (Nidhi 2015).

Large numbers of techniques have been proposed in the past for object detection, tracking and classification. The moving object detection algorithm with simple background subtraction has been proposed (Sainadh and Arvind 2015). The real time recognition of vehicles and pedestrian from videos using background and edge-based subtraction has proposed (Gokul, Santosh Kumar and Sreeraj 2012). The detection of pedestrians in the video by using motion and shape information has proposed (Ray et al. 2016). The moving object trajectories are generated for indoor space has been proposed (Ryoo et al. 2016). The features extraction of moving objects in a video and clustering the motion trajectories by using hierarchical clustering has been proposed (Buzan, Sclaroff and Kollios 2004). The real time detection and tracking of the objects in the videos and classification using clustering has been proposed (Ray and Miao 2016).

There are various types of object tracking and classification techniques using a neural network. Convolutional Neural Network (CNN) has been extensively used for pattern recognition and image recognition for moving objects in the video. The real time object recognition model by using deep convolution neural network to extract deep features has been proposed (Yang, Wang and Wu 2018). The detection of fast moving vehicles by using convolutional neural network has been proposed (Zhu et al. 2017). A multi-level three-dimensional convolutional neural network for moving objects recognition has been proposed (He et al. 2017).

Recurrent Neural Network (RNN) is another technique for tracking the objects that can determine the further activities based on the current. The online multi-target tracking using recurrent Networks (Milan et al. 2016) tracks the objects based on tracking-by-detection. The unused data in a video sequence are 
discarded and reduced small measurement per frame then associate each measurement to the corresponding target. It has two main blocks: state prediction for predicting target motion and update to correct the state distribution with task management and data association. In our approach, the object tracking is based on the frame by frame detection. This model tracks the moving objects collected as a batch of videos. The large number of moving objects can be tracked using the multi-motion-based detection method

The trajectory-based anomalies behavior detection using k-means clustering and Hidden Markov Model (HMM) has been proposed (Cai et al. 2015). An unsupervised clustering method for anomaly detection of the moving objects has been proposed (Ranjith et al. 2015). More existing methods for pattern recognition of moving objects are using unsupervised learning methods. This paper proposes an unsupervised clustering algorithm and supervised learning method for pattern recognition then comparing between them.

\section{PROPOSED METHOD}

The proposed method recommends multiple moving objects tracking and pattern recognition. This method uses motion-based techniques for the tracking of moving objects. The spectral Clustering algorithm and Naive Bayes are used to recognize the pattern of moving objects.

\subsection{Object Tracking Model}

Object tracking system is the process of tracking the moving object and identifying the direction of their motion. The system processes a given video to find multiple moving objects in it. In order to work out this model flawlessly, the video stream needs to be largely static over consecutive frames of a video. The substantial changes between two successive frames are counted as moving objects. The fundamental concept of Gaussian Mixture Model-based foreground and background segmentation is used. The model further does its own calculations and analysis to keep track of identified motions. This model tracks multiple moving objects based on motion. The proposed model is explained in Figure 1.

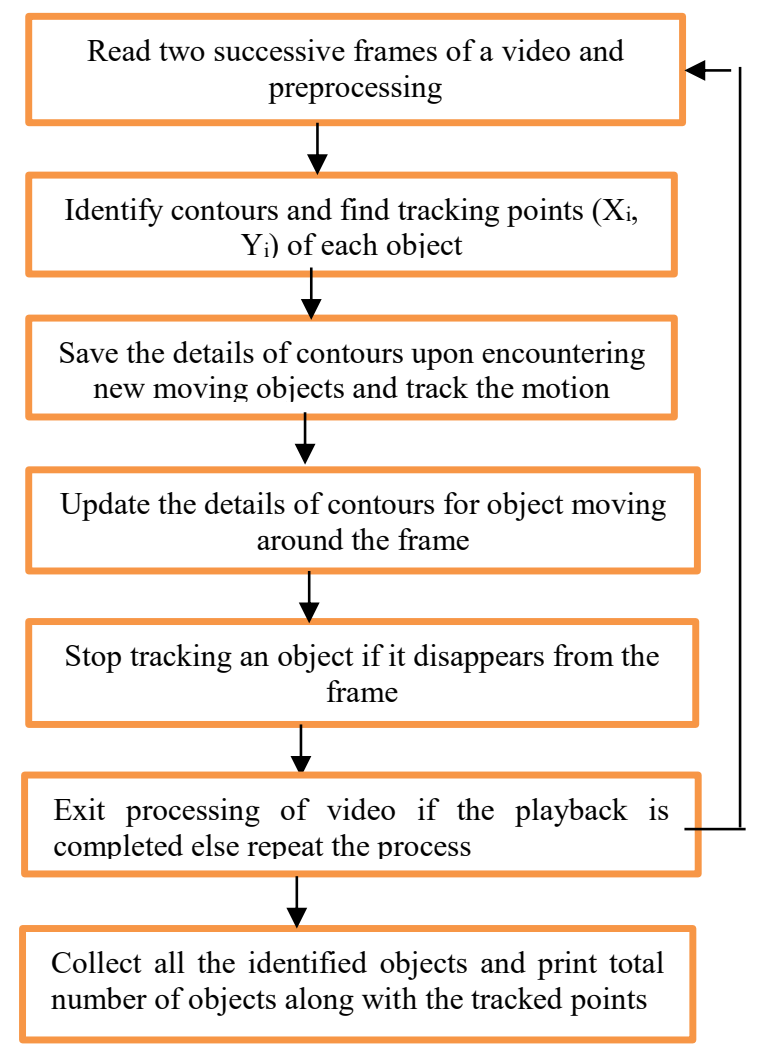

Figure 1: Object tracking model. 


\subsubsection{Read two successive frames of a video and preprocessing}

This model focuses on identifying objects that are moving in a video. In this step, the sequence of frames is read from a video. The main algorithm for detecting a moving object and tracking its path is computing frame difference of two consecutive frames in a video. The videos are expected to be shot static either by CCTV or using fixed camera stands.

After capturing frame difference, the result is optimized so that the detection becomes easier. In this process, appropriate threshold values are imposed, and noises are removed as far as possible. The noise reduction parameters depending on the video, so appropriate trials should be taken to find the best result.

\section{Pseudocode}

1. Frame1=video.read ()

2. Frame2=video.next()

3. Diff=absdiff(frame1, frame2)

\subsubsection{Identifying contours and finding tracking points}

Upon getting remarkable changes in the consecutive frames, the next process is contour detection. A contour is a collection of pixels of a frame which is identified by the OpenCV library. There will be zero, one or more contours depending upon the motion inside the frames. If the contours are non-zero and the size of a contour is at least equal to or greater than the specified min-area, the centers of each contour is identified for further analysis.

\section{Pseudocode}

1. Contours $=$ findContours $($ diff $)$

2. If Contours $>0$ and area(Contours) $>=$ min-area

3. Center=findCenter(contours)

\subsubsection{Save the details of contours upon encountering new moving objects}

If there are initially no objects in the frame or some new objects appear into the frame, those will be tracked, and their details are saved. The details of a contour have properties such as ID, contour shape and points of the contour motion path. These properties will be helpful in identifying the same object throughout the frame. The points of contour motion path will be accumulated and will be used in recognizing the object's movement pattern. The identified moving objects are enclosed with the bounding box and shown on the output video window so that the viewer gets the perception of objects being tracked.

\section{Pseudocode}

1. If contours have new objects

2. Contour class $=$ contour (id, contour_shape, centre_points)

\subsubsection{Update the details of contours for objects moving around the frame}

If the same object moves inside the frame, it is necessary to keep the same instance of the moving object. For this, the object's motion and points are updated. The same object is identified by its previous position and comparing the distance between the previous position and current position with the specified minimum threshold.

\section{Pseudocode}

1. If contours have same objects 
2. Contour_class $=$ updateContour (id, centre_point)

\subsubsection{Stop tracking an object if it disappears from the frame}

If an object moves out of the frame or disappears, the object should be stopped tracking and its properties should be finalized before disposing. The disappearance of an object can be identified by calculating the difference between previously tracked total objects and current tracked total objects. If current objects are less than the previous one, object(s) are considered as exited from a frame.

\section{Pseudocode}

1. If contours disappeared

2. Save contour_class to tracked_objects

3. Delete (contour_class)

\subsubsection{Identify total objects and print its tracked points}

When the scanning of the video is completed, the objects and its points can be calculated. While the previous processing was happening repetitively frame by frame, the respective objects and its tracked points were stored. After the processing is finished, the total points can be calculated accurately. Similarly, their motion path points can also be printed on the console output.

\section{Pseudocode}

1. Tracked the objects

2. Print object points

3. Print total number of tracked objects

\subsection{Pattern Recognition}

Spectral clustering is a type of supervised learning that is used to cluster the set of unlabeled data. It is a technique to cluster the data by making the use of the spectrum of the similarity matrix of the data and perform dimensionality reduction in fewer dimension before clustering. The goal of spectral clustering is to cluster data that is connected but not necessarily compact or clustered within convex boundaries. The DTW algorithm is used before the spectral clustering. DTW is one of the algorithms that calculates the similarity between the two-time series and finding the optimal global alignment between two-time series (Jingyi et al. 2016).

\section{Pseudocode of Trajectory Clustering}

1. Divide the two trajectories into equal number of points.

2. Calculate the Euclidean distance between the first point in the first trajectory and every point in the second trajectory. Store the minimum distance calculated. (this is the 'time warp' stage)

3. Move to the second point and repeat step 2. Move step by step along points and repeat 2 till all points are exhausted.

4. Repeat step 2 and step 3 but with the second trajectory as a reference point.

5. Repeat step 1, step 2 and step 3 for $n$ trajectories

6. Add up all the minimum distances that were stored and this is a true measure of similarity between the trajectories

7. Input similarity matrix of step 6 into the spectral clustering algorithm

8. Get the trajectories into normal and abnormal class

In our proposed model, first, DTW is used to find out the similarities between any two curves. Then, the distance matrix calculated using DTW is used as input for spectral clustering to cluster the normal and abnormal trajectory. 
Naïve Bayes is a powerful algorithm for classification and prediction. It works for a dataset with millions of records. It gives greater results for textual and image data analysis such as natural language processing. It is easy and fast to predict the class of test data set. It also performs well in multiclass prediction. When the assumption of independence holds, a Naive Bayes classifier performs better compared to other models like logistic regression. It is a very powerful tool for trajectory data classification. We have a trajectory data set $\mathrm{T} 1, \mathrm{~T} 2, \mathrm{~T} 3, \ldots, \mathrm{Tn}$ and each trajectory has data $(\mathrm{x} 1, \mathrm{y} 1),(\mathrm{x} 2, \mathrm{y} 2), \ldots,(\mathrm{xn}, \mathrm{yn})$. The trajectory data sets are the path of tracked vehicles from the videos of YouTube. In this paper, Naïve Bayes is used for developing the model to classify the trajectories into normal and abnormal behavior. The features such as slope between each two-point, average slope and standard deviation are the feature extraction of each trajectory. The accuracy of a model is calculated by using the confusion matrix. The confusion matrix is represented in Table1.

Table 1: Confusion Matrix.

\begin{tabular}{|l|l|l|l|}
\hline \multicolumn{2}{|c|}{} & \multicolumn{2}{|c|}{ Actual } \\
\cline { 3 - 4 } \multicolumn{2}{|c|}{ Positive } & Negative \\
\hline \multirow{3}{*}{ Predicted } & Positive & A: True positive & B: False Positive \\
\cline { 2 - 4 } & Negative & C: False Negative & D: True Negative \\
\hline
\end{tabular}

The True Positive refers to correctly identified instance and True Negative refers to correctly rejected instances. The False Positive represents incorrectly identified instances and False Negative refers to incorrectly rejected instances. Accuracy, as an evaluation criterion for classification approaches, is calculated for a given dataset based on the percentage of the data which are correctly classified by the total number of predictions. In classification methods, accuracy also represents how well a classifier can correctly classify the instances. The equation below can be employed to compute accuracy of a classifier The accuracy $(\mathrm{AC})$ is determined by the proposition of the total number of predictions that were correct. It is calculated by:

$$
\mathrm{AC}=(\mathrm{A}+\mathrm{D}) /(\mathrm{A}+\mathrm{B}+\mathrm{C}+\mathrm{D}) .
$$

\section{EXPERIMENTS AND RESULTS}

The proposed model that was implemented in a machine with Windows 10 operating system. It was implemented and tested on Intel Core I5 CPU @2.3GHz with 8GB RAM. The programming platform for tracking the objects was Python 3.6 and the OpenCV library. The Python with Jupiter programming environment was used for pattern recognition. The proposed model used varieties of Python packages such as Numpy, Pandas, matplotlib, dtaidistance, Sklearn and Keras. The overall results are divided into object tracking result and pattern recognition result.

\subsection{Object Tracking Results}

Object tracking system can use any types of videos stored in database or social media or can create new videos. In this experiment, the YouTube videos were collected, and tracking was done in real time to test this system. Most of the videos for testing the system were real videos of social networking sites. We prepared some videos in a real street and parking lots where the vehicles are moving. The online tracking is shown in Figure 2 to Figure 7. Each figure shows the moving objects at a time and rectangle around objects shows travelling of motion of objects. 


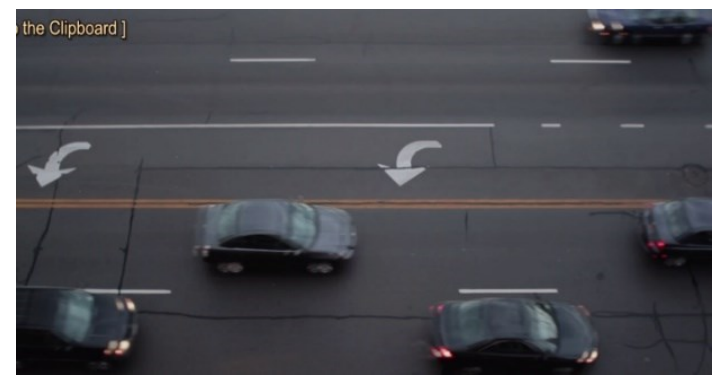

Figure 2: Sample 1 video.

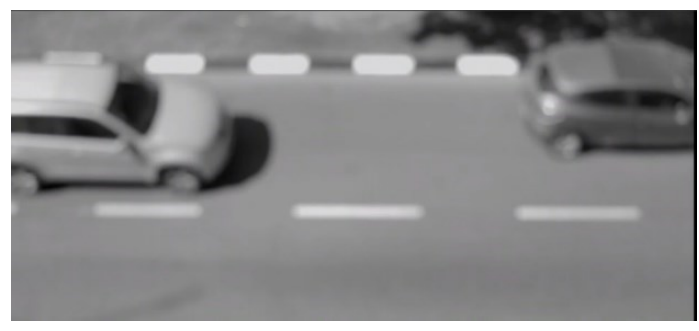

Figure 4: Sample 2 video.

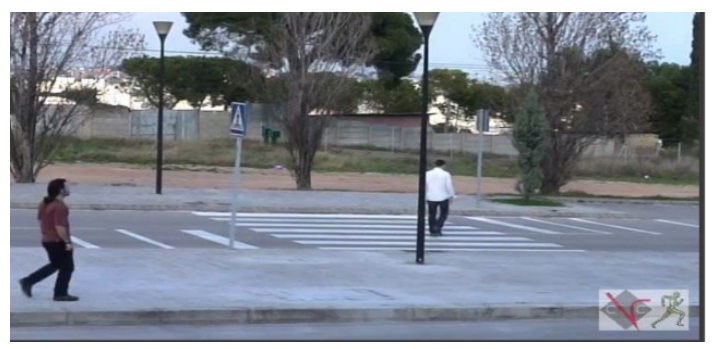

Figure 6: Sample 3 video.

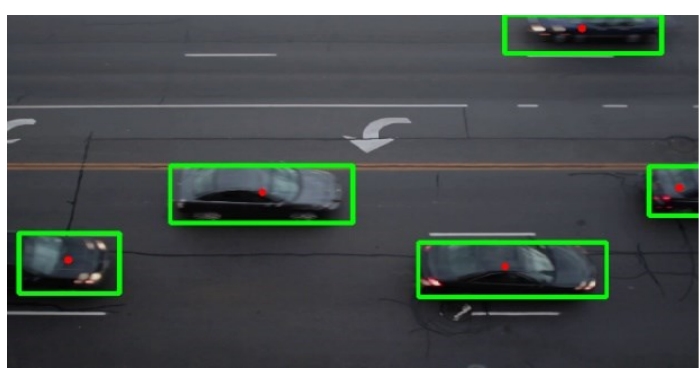

Figure 3: Tracking of sample 1 video.

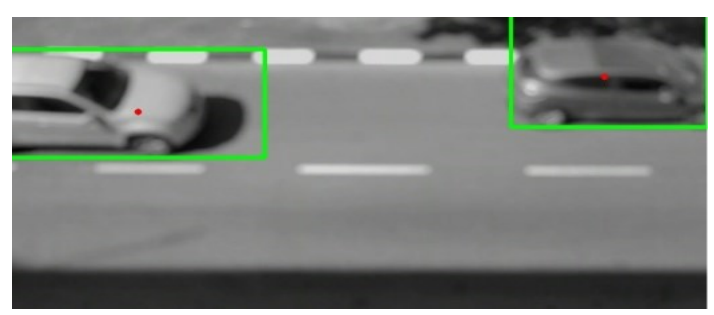

Figure 5: Tracking of sample 2 video.

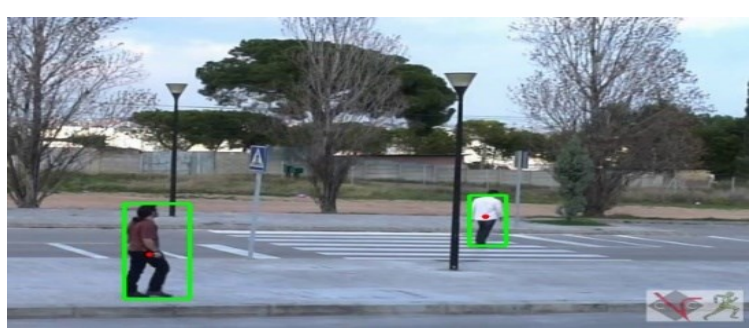

Figure 7: Tracking of sample 3 video.

By using experimental study, we verify our tracking model by finding tracking rate, recall and precision. Table 2 gives the True Positive (TP), False Positive (FP) and False Negative (FN) values of standard real time videos and finding the recall and precision (Gokul, Santosh Kumar and Shreeraj 2012).

$\mathrm{TP}=$ Tracked the actual object

$\mathrm{FP}=$ Tracked the object that do not correspond to actual object

$\mathrm{FN}=$ Object not tracked

Tracking rate $=(\mathrm{TP} * 100) /(\mathrm{TP}+\mathrm{FN})$

Recall $=\mathrm{TP} /(\mathrm{TP}+\mathrm{FN})$

Precision $=\mathrm{TP} /(\mathrm{TP}+\mathrm{FP})$

Table 2: Recall and precision of different videos.

\begin{tabular}{|c|c|c|c|c|c|}
\hline Based on objects in videos & TP & FP & FN & Recall & Precision \\
\hline Number of Vehicles only & 115 & 3 & 4 & 0.96 & 0.97 \\
\hline Number of Pedestrian only & 82 & 1 & 3 & 0.96 & 0.98 \\
\hline Number of Vehicles and pedestrians & 96 & 2 & 3 & 0.96 & 0.97 \\
\hline
\end{tabular}


From Table 2, we found that the proposed tracking model has good performance because the recall and precision value are more than $95 \%$. The tracking has very good performance if the distance between the objects is far and motion is medium (not very slow and not very fast).

\subsection{Pattern Recognition Results}

In this experiment, pattern recognition for moving vehicles can be performed by Spectral clustering algorithm. When running the object tracking system, the data set for each object are generated. These data set are analyzed by writing a code in Python. The unsupervised machine learning algorithm Spectral clustering is used to find abnormal and normal activities of the vehicles that is stored in a database or videos. The sample result is demonstrated in Figure 8.

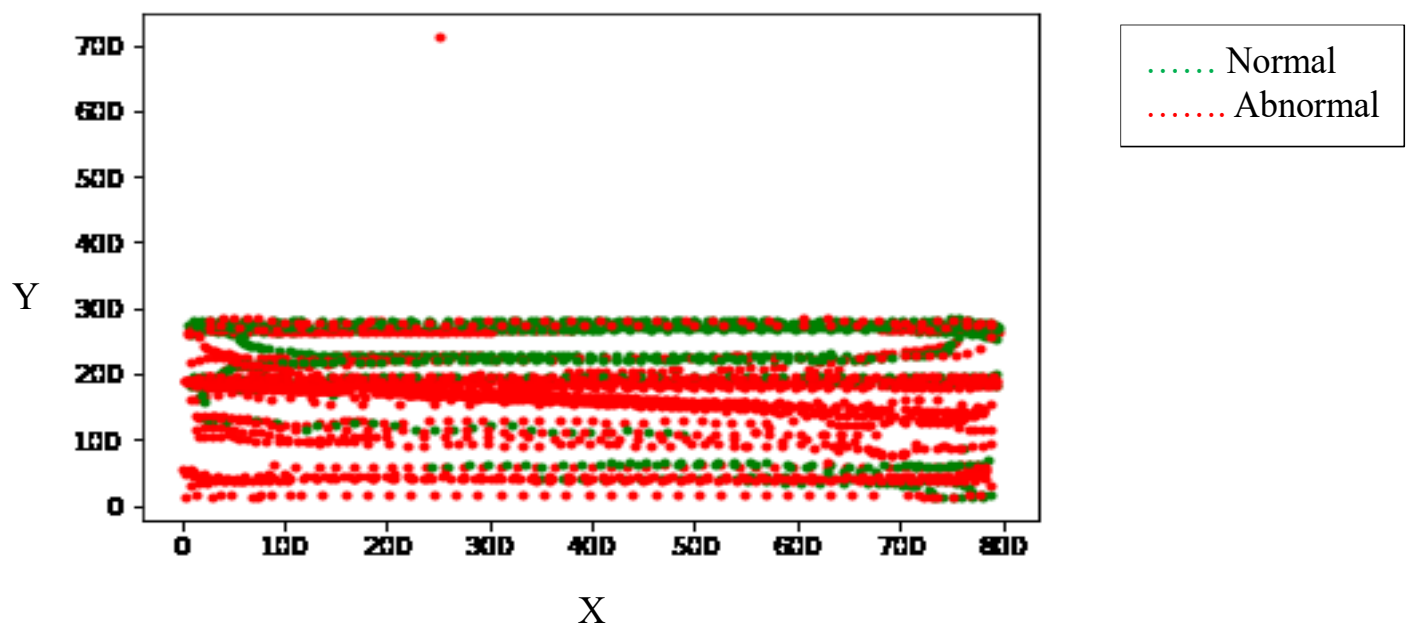

Figure 8: Clustering sample result.

Figure 8 is a representation of the result from spectral clustering of 60 moving vehicles collected from YouTube videos. This figure divides the trajectories into two groups which are represented in the green color cluster and red color cluster. The vehicles with normal behaviors are represented as green color and vehicles with abnormal behaviors are represented as red color. If the cars are moving in a straight line, it is normal behavior otherwise abnormal behavior. This type of pattern recognition technique is only suited in straight roads and parking areas. In parking areas, more accidents are happened because of abnormal activities and violating the rules.

Naïve Bayes algorithm is used for developing and testing the accuracy of a model. We divide the data set into $80 \%$ training set and $20 \%$ testing set. The accuracy and running time of the models are explained in Table 3 and Table 4.

Table 3: Accuracy and running time of 12 trajectories (20\% of total trajectories).

\begin{tabular}{|c|c|c|}
\hline Model & Accuracy (\%) & $\begin{array}{c}\text { Algorithm running time } \\
\text { (milliseconds) }\end{array}$ \\
\hline Spectral Clustering & 70.0 & 8.2 \\
\hline Naïve Bayes & 81.2 & 0.9 \\
\hline
\end{tabular}


Table 4: Accuracy and running time of 60 trajectories (100\% trajectories).

\begin{tabular}{|c|c|c|}
\hline Model & Accuracy (\%) & $\begin{array}{c}\text { Algorithm running time } \\
\text { (milliseconds) }\end{array}$ \\
\hline Spectral Clustering & 78.1 & 62.4 \\
\hline Naïve Bayes & 86.0 & 10.2 \\
\hline
\end{tabular}

Table 3 and Table 4 show the result of an experiment. The accuracy of the proposed method was calculated by using confusion matrix which was described in Table 1 . The accuracy of trajectory clustering method is around $78.1 \%$ and the time to create the clusters is 62.4 milliseconds while considering 60 trajectories that is shown in Table 4. Similarly, The accuracy of trajectory clustering method is around $70.0 \%$ and the time to create the clusters is 8.2 milliseconds while considering 12 trajectories (testing set $20 \%$ of total trajectories) that is shown in Table 3. Spectral clustering does not require training, and trajectories collection is very simple with minimum data and low dimensionality. The accuracy of Naïve Bayes method is around $86.0 \%$ and running time is 10.2 milliseconds while considering 60 trajectories that is shown in Table 4. Similarly, The accuracy of Naïve Bayes method is around $81.2 \%$ and the time to create the clusters is 0.9 milliseconds while considering 12 trajectories (testing set $20 \%$ of total trajectories) that is shown in Table 3. Therefore, the result shows that Naïve Bayes has higher accuracy than trajectory clustering method and the algorithm running time of Naïve Bayes is faster than trajectory clustering.

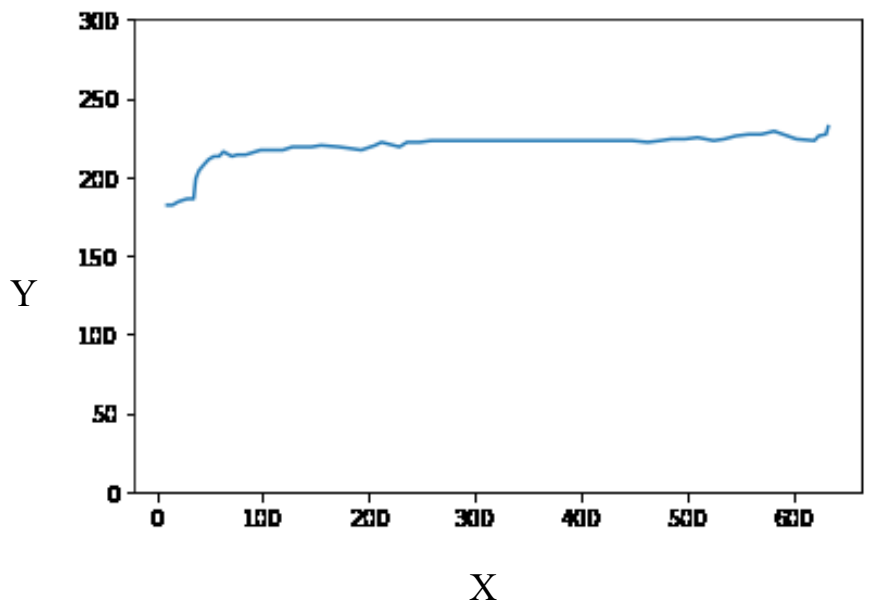

Figure 9: Example of Abnormal Motion.

When the model using Naïve Bayes is tested by considering a trajectory, the detection is more accurate. The trajectories are tested to determine the accuracy of our model. Figure 9 represents a sample of abnormal trajectory. This sample is tested by using our model and the result shows that the trajectory is abnormal. while calculating the accuracy, the detection is considered as true negative in confusion matrix. The trajectory looks like a straight line is considered as normal and the curve line like figure 9 is considered as abnormal.

\section{CONCLUSIONS}

This paper presented a model for real time object tracking and scalable pattern recognition of moving objects that can process the stored videos in a database or social networks. This approach used multi-object 
motion detection techniques for object tracking, and spectral clustering and Naïve Bayes for pattern recognition with a goal of building a scalable system that can be used to analyse large numbers of videos existing in social networking website or large multi-media database. We found the tracking model is fast and accurate with high recall and precision values in our tested data. The objects were tracked perfectly which was evident during multimedia streaming are shown in object tracking figures. This new method used spectral clustering unsupervised learning and Naïve Bayes supervised learning for classifying the trajectories of moving objects. The result shows that Naïve Bayes is more accurate than the spectral clustering method for pattern recognition. Similarly, the average timing of Naïve Bayes is dramatically faster than clustering method. The novel part of this work is scalability. The tracking rate of the objects is high and pattern recognition is very fast which is evident from Table 3 and Table 4 . The limitation of this model is the limited size of tested videos and different scenarios of moving multiple objects. In our future work we plan to test scalability of pattern recognition for much larger of video files. We also plan for the improvement of clustering algorithm and using more supervised learning methods for classification of trajectory.

\section{REFERENCES}

Abhari, A., S. Banihashemi, and J. Li. 2017. "Object Movement Detection by Real-Time Deep Learning for Security Surveillance Camera”. IEEE Conference of Computer Society, pp. 484-448.

Buzan, D., S. Sclaroff, and G. Kollios. 2004. "Extraction and Clustering of Motion Trajectories in Video". Proceedings of the $17^{\text {th }}$ International Conference of Pattern Recognition, pp. 520-524, doi:10.1109/ICPR.2004.1334287.

Cai, Y., H. Wang, X. Chen and H. Jiang. 2015. "Trajectory-based anomalous Behavior Detection for Intelligent Traffic Surveillance". IET Intelligent Transport Systems, vol. 9, pp. 810-816

Gokul, S., G. Santosh Kumar, and M. Sreeraj. 2012. "Real Time Recognition of Pedestrian and Vehicles from Videos". ACM International Conference Proceedings Series, pp. 517-523

He, T., H. Mao, and Z. Yi. 2017. "Moving Object Recognition using Multi-View Three-dimensional Convolutional Neural Networks". Neural Computing and Applications, vol. 28, pp. 3827-3835. doi:10.1007/s00521-016-2277-9

Jingyi, S., Z. Dongyang, H. Weiping and L. Jun, 2016. "A Novel Similarity Measure Approach for Time Series Based on PLA and DTD”. 35 ${ }^{\text {th }}$ Chinese Control Conference, pp. 7159-7163, doi: 10.1109/ChiCC.2016.7554488

Mazimpaka J. D., and S. Timpf. 2016. "Trajectory Data Mining: A Review of Methods and Applications". Journal of Spatial Information Science, vol. 13, pp. 61-99.

Milan, A., S.H. Rezatofighi, A. Dick, I. Reid, and K. Schindler. 2016. "Online Multi-target Tracking using Recurrent Neural Networks". Retrieved from: https://www.aaai.org/ocs/index.php/AAAI/AAAI17/ Paper/viewpaper/14184 .

Nidhi, 2015. "Image Processing and Object Detection”. International Journal of Applied Research, vol. 1, pp. 396-399

Pedro, M. R., J. R. Antonio, and O. J. Armand. 2007. “Color-space and Color Segmentation for Real Time Object Recognition in Robotics Application”. Revista do detua, vol. 4, pp. 1-6

Ranjith, R., J. J. Athanesious and V. Vaidehi. 2015. "Anomaly detection using DBSCAN Clustering Technique for Traffic Video Surveillance". Seventh International Conference on Advanced Computing (ICoAC), pp. 1-6, doi: 10.1109/ICoAC.2015.7562795

Ray, L., and T. Miao. 2016. "Towards Real-time Detection, Tracking and Classification of natural video". Proceedings of the $13^{\text {th }}$ IEEE conference on Computer and Robot Vision, pp. 236-241, doi:10.1109/CRV.2016.23 
Ryoo, H., S. Kim, T. Kim, D. Seo, and K. Li. 2016. "Generation of Moving Object Trajectories in Indoor Space". Proceedings of the Eighth ACM SIGSPATIAL International Workshop on Indoor Spatial Awareness, pp. 43-46

Sainadh, B., and S. Arvind. 2015. “An Advanced Object Detection using K-means Clustering”. IJESC, pp. $1528-1531$

Xu, J., G. Ye, G. Herman, and B. Zhang. 2008. “An Efficient Approach to Detecting Pedestrians in Video". Proceedings of the 16th ACM international conference on Multimedia, pp. 789-792

Yang, H., L. Shao, F. Zheng, L. Wang, and Z. Song. 2011. "Recent advances and trends in visual tracking: A Review". Neurocomputing, vol. 74, pp. 3823-3831. doi: 10.1016/j.neucom.2011.07.024

Yang, L., L. Wang, and S. Wu. 2018. "Real-time Object Recognition Algorithm Based on Deep Convolutional Neural Network". Proceedings of the $3^{\text {rd }}$ IEEE conference on cloud computing and big data analysis, pp. 331-335, doi:10.1109/ICCCBDA.2018.8386537

Yilmaz, A., O. Javed, and M. Shah. 2006. "Object tracking A Survey”. ACM Computing Surveys (CSUR), vol. 38, pp. 1-4

Zalik, K. R. 2008. “An Efficient K-means Clustering Algorithm”. Pattern Recognition Letters, vol. 29, pp. 1385-1391, doi: 10.1016/j.patrec.2008.02.014

Zhang, Y., and L. Wu. 2012. "Classification of Fruits using Computer Vision and a Multiclass Support Vector Machine”. Sensors (Basel, Switzerland), vol. 12, pp. 12489-12505.doi:10.3390/s120912489

Zhu, F., Y. Lu, N. Ying, and G. Giakos. 2017. "Fast Vehicle Detection Based on Evolving Convolutional Neural Network". Proceedings of the IEEE conference of Instrumentation and Measurement Society, pp. 1-4. doi: 10.1109/IST.2017.8261505

\section{AUTHOR BIOGRAPHIES}

DIPAK PUDASAINI is a PhD student in the Department of Computer Science at Ryerson University and Assistant Professor at Tribhuvan University, Nepal. He holds a M. Sc. in Computer Science from Ryerson University. He is a computer Science subject committee member of Tribhuvan University. His research interests include data science and web service selection. His email address is dpudasaini@ryerson.ca

ABDOLREZA ABHARI is a Professor in the Department of Computer Science at Ryerson University and director of DSMP lab (http://dsmp.ryerson.ca). He holds a Ph.D. in Computer Science from Carleton University. His research interests include web social networks, data science, AI and agent systems, network simulation, and distributed systems. His email address is aabhari@ryerson.ca 